INPLASY

PROTOCOL

To cite: Lin et al. The role of Central nervous system (CNS) prophylaxis in preventing DLBCL patients from CNS relapse: a network metaanalysis. Inplasy protocol 2021120131. doi:

10.37766/inplasy2021.12.0131

Received: 29 December 2021

Published: 29 December 2021

Corresponding author:

Zhijuan Lin

zjuan_lin@126.com

Author Affiliation:

The first affiliated hospital of

Xiamen hospital.

Support: National Natural

Science Found.

Review Stage at time of this submission: Data analysis Completed but not published.

Conflicts of interest:

None declared.

\section{The role of Central nervous system (CNS) prophylaxis in preventing DLBCL patients from CNS relapse: a network meta-analysis}

Lin, ZJ1; Chen, X2; Liu, L3; Zeng, HY4; Li, ZF5; Xu, B6.

Review question / Objective: Our analysis aimed to answer the following questions: (1) the role of rituximab in reducing the risk of CNS relapse; (2) the optimal prophylactic strategies (intravenous chemotherapy, intrathecal injections or novel target agents) to prevent CNS occurrence in patients with high CNS relapse risk in rituximab era; and (3) the optimal timing of HD-MTX CNS prophylaxis and its preventive efficacy as well as toxicity.

Condition being studied: Secondary central nervous system (CNS) relapses are an uncommon yet devastating complication in diffuse large B cell lymphoma (DLBCL). Although several prophylaxis attempts were employed clinically in order to reduce the CNS relapse rate, the optimal management remained uncertain.

INPLASY registration number: This protocol was registered with the International Platform of Registered Systematic Review and Meta-Analysis Protocols (INPLASY) on 29 December 2021 and was last updated on 29 December 2021 (registration number INPLASY2021120131).

\section{INTRODUCTION}

Review question / Objective: Our analysis aimed to answer the following questions: (1) the role of rituximab in reducing the risk of CNS relapse; (2) the optimal prophylactic strategies (intravenous chemotherapy, intrathecal injections or novel target agents) to prevent CNS occurrence in patients with high CNS relapse risk in rituximab era; and (3) the optimal timing of HD-MTX CNS prophylaxis and its preventive efficacy as well as toxicity.

Condition being studied: Secondary central nervous system (CNS) relapses are an uncommon yet devastating complication in diffuse large B cell lymphoma (DLBCL). 
Although several prophylaxis attempts were employed clinically in order to reduce the CNS relapse rate, the optimal management remained uncertain.

\section{METHODS}

Participant or population: The impact of different interventions on CNS relapse rate in DLBCL patients without CNS involvement at diagnosis.

Intervention: All CNS prophylaxis interventions.

Comparator: All CNS prophylaxis interventions.

Study designs to be included: All prospective, randomized controlled trials (RCTs), clinical trials, cohort studies and case-control studies.

Eligibility criteria: These trials should be published in English language from 2000 to 2021. Considering the low incidence of secondary CNS lymphoma, the total number of patients in the studies should be greater than 100 in order to avoid bias.

Information sources: We used the MEDLINE, Embase, and Cochrane Library databases to search the RCTs met our selection criteria. We also searched clinicalTrials.gov and the international clinical trial registry platform for completed and ongoing trials. In addition, we searched abstracts that published on American Society of Hematology (ASH), The European Hematology Association (EHA) or American Society of Clinical Oncology (ASCO) meetings. The searches were restricted in English since January 2000.

Main outcome(s): The primary outcome in our study was CNS relapse rate of different interventions.

Quality assessment / Risk of bias analysis: We used the Cochrane Collaboration's tool to assess the quality of selected RCTs and Newcastle-Ottawa Scale (NOS) assessment to assess the quality of selected cohort studies or case-control studies.

Strategy of data synthesis: Conventional meta-analysis and network meta-analysis.

Subgroup analysis: NA.

Sensitivity analysis: NA.

Language: English.

Country(ies) involved: China.

Keywords: central nervous system (CNS) relapses, diffuse large $B$ cell lymphoma (DLBCL), prophylaxis.

Contributions of each author:

Author 1 - Zhijuan Lin - ZJL involved in data collection along with the risk of bias assessment strategy. ZJL drafted the manuscript.

Email: zjuan_lin@126.com

Author 2 - Xing Chen - XC involved in data collection along with the risk of bias assessment strategy.

Email: chenxingpku@163.com

Author 3 - Long Liu - LL provided statistical expertise.

Email: kucangyaoren@126.com

Author 4 - Hanyan Zeng - HYZ contributed to the development of the selection criteria. Email: pinkhyz@126.com

Author 5 - Zhifeng Li - ZFL took part in conception and design, and contributed to the development of the selection criteria.

Author 6 - Bing Xu - BX took part in conception and design. 